\title{
Efficacy and Safety of Using Amplatzer Ductal Occluder for Transcatheter Closure of Perimembranous Ventricular Septal Defect in Pediatrics
}

\author{
Mehdi Ghaderian ${ }^{1, *}$; Mahmood Merajie ${ }^{2}$; Hodjjat Mortezaeian ${ }^{2}$; Moghadam Aarabi ${ }^{2}$; \\ Yoosef Mohammad $^{2}$; Akbar Shah Mohammadi $^{2}$ \\ ${ }_{1}^{1}$ Department of Pediatric Cardiology, Child Growth and Development Research Center, Isfahan University of Medical Sciences, Isfahan, IR Iran \\ 2 Pediatric Cardiology Department, Shaheed Rajaiee Cardiovascular Research Center, Iran University of Medical Sciences, Tehran, IR Iran \\ *Corresponding author: Mehdi Ghaderian, Department of Pediatric Cardiology, Child Growth and Development Research Center, Isfahan University of Medical Sciences, Isfahan, IR \\ Iran.E-mail: ghader_45@yahoo.co.uk \\ Received: June 27, 2014; Accepted: January 5, 2015
}

\begin{abstract}
Background:Perimembranous VentricularSeptal Defect(PMVSD) is the mostcommon subtype of ventricular septal defects. Transcatheter closure of PMVSD is a challenging procedure in management of moderate or large defects.

Objectives:The purpose of this study was to show that transcatheter closure of perimembranous ventricular septal defect with Amplatzer Ductal Occluder (ADO) is an effective and safe method.

Patients and Methods: Between April 2012 and April 2013, 28 patients underwent percutaneous closure of PMVSD using ADO. After obtaining the size of VSD from the ventriculogram a device at least $2 \mathrm{~mm}$ larger than the narrowest diameter of VSD at right ventricular side was chosen. The device deployed after confirmation of its good position by echocardiography and left ventriculography. Follow up evaluations were done 1 month, 6 months, 12 months and yearly after discharge with transthoracic echocardiography and 12 lead electrocardiography.

Results: The mean age of patients at procedure was $4.7 \pm 6.3$ (range 2 to 14 ) years, mean weight $14.7 \pm 10.5$ (range 10 to 40 ) kg. The mean defect size of the right ventricular side was $4.5 \pm 1.6 \mathrm{~mm}$. The average device size used was $7.3 \pm 3.2 \mathrm{~mm}$ (range 4 to $12 \mathrm{~mm}$ ). The ADOs were successfully implanted in all patients. The VSD occlusion rate was $65.7 \%$ at completion of the procedure, rising up to $79.5 \%$ at discharge and 96.4\% during follow-up. Small residual shunts were seen at completion of the procedure, but they disappeared during follow-up in all but one patient. The mean follow-up period was $8.3 \pm 3.6$ months (range 1 to 18 months). Complete atrioventricular block (CAVB), major complication or death was not observed in our study.

Conclusions: Transcatheter closure of PMVSD with ADO in children is a safe and effective treatment associated with excellent success and closure rates, but long-term follow-up in a large number of patients would be warranted.
\end{abstract}

Keywords: Ventricular Septal Defect; CAVB Protocol; Septal Occluder Device

\section{Background}

Among congenital heart defects (CHD), Ventricular septal defect (VSD) is the most common form. VSD approximately constitutes for $30 \%$ of congenital heart diseases and by newer echocardiography and experience of echocardiographers now the recognition of VSDs is increased up to 50/1000 live births and bigger rates in human fetuses (1-4). Perimembranous ventricular septal defect (PMVSD) is the most common subtype of this defect and responsible for more than 85\% of VSDs. Small VSDs (less than one third of aortic valve size) do not cause significant pulmonary overload or overcirculation and do not increase pulmonary/systemic blood flow (QP/QS) significantly. Medical treatment, surgical therapy or interventional closure of these defects is not suggested and the outcome of these patients with these small defects is very good. In cases with increased pulmonary flow and moderate or larger defects surgical or interventional ap- proach is recommended. Compared to surgical approach with a history of 60 years' experience, transcatheter closure of PMVSD is a newer procedure. Because of the shorter hospitalization time and not needing cardiopulmonary bypass and sternotomy, transcatheter approach is recommended in some types of VSD. Amplatzer for VSD closure was added to interventional management before 2000 for the first time and the Amplatzer Membranous VSD Occluder for closing of membranouse defects was first described after 2000 and added to muscular VSD Occluder (5-7). The Ampltzer perimembranous ventricular septal defect occluder (APMVSDO) has shown to be effective in closing PMVSD in several papers $(8,9)$. One of the most serious complications of this method is high incidence of atrioventricular conduction block (AVB) (10-13). Comparing surgical closure and percutaneouse closure of isolated VSD, early and delayed atrioventricular block

Copyright (C) 2015, Growth \& Development Research Center. This is an open-access article distributed under the terms of the Creative Commons Attribution-NonCommercial 4.0 International License (http://creativecommons.org/licenses/by-nc/4.0/) which permits copy and redistribute the material just in noncommercial usages, provided the original work is properly cited. 
was described in less than $1 \%$ of patients after surgical closure, whereas this complication ranges from 3\% to $20 \%$ after percutaneous closure $(12,14)$. The proximity of the His bundle and the right ventricular disc of the APMVSDO and the squeezing effect of both discs and inducing of fibrous tissue formation during the time by device implantation may cause complete atrioventricular block (CAVB) after transcatheter closure of PMVSD by using an APMVSDO.

\section{Objectives}

The purpose of this study was to evaluate the results of our experience in early and mid-term follow up of transcatheter closure of VSD using Amplatzer ductal occluder (ADO) in children because it has shorter left ventricular rim and no right ventricular disc and no squeezing effect and therefore, it is supposed to have lower incidence of CAVB.

\section{Patients and Methods}

The study protocol was approved by the Ethics Committee of the Iran University of Medical Sciences in Iran. In this study all patients who had undergone transcatheter closure of VSD using ADO between April 2012 and April 2013 in Shaheed Rajaee Cardiovascular Medical and Research Center were included. Parental consent was obtained for this study. In this period of time $28 \mathrm{pa}$ tients underwent transcatheter closure of PMVSD using ADO. Patients were examined by a standard echocardiographic protocol and experienced operator; all patients underwent transthoracic echocardiography (TTE) that was performed with a GE Vivid 3 machine including Mmode, two-dimensional and color Doppler. The standard technique was used to obtain the measurements in a non-sedated for older children and sedated for younger children in quiet and wakeful state. Size and sort of VSD were examined in different standards four chambers, five chambers and parasternal in short and long-axis views. Patients with clinical or echocardiographic evidence of significant left to right shunt whose Qp/Qsws was $\geq 1.5$ due to isolated PMVSD, were selected and included in the study. A significant left-to-right shunt was considered to be a perimembranous ventricular septal defect and the following data were found: (a) prominent cardiomegaly and lung congestion on standard chest X-ray; (b) left atrial to aortic ratio $>1.5$ in long axis parasternal view examination by echocardiography defined as left atrial enlargement; (c) left ventricular overload and enlargement in echocardiography; (d) frequent respiratory in fections in past medical history (six events during a year) and/or failure to thrive; (e) Infants selected for transcatheter VSD closure that weighed $\geq 10 \mathrm{~kg}$. Exclusion criteria were (a) VSD associated with any other congenital heart disease which had to be corrected by surgical approach; (b) patients with significant non-cardiac and cardiac comorbidities and abnormalities that could impact clinical outcome of VSD closure; (c) VSD with severe pulmonary artery hypertension (Eisenmenger syndrome) and right to left shunt or pulmonary vascular resistance of greater than 8 Woods units; (d) patients with perimembranous VSD and prolapse of an aortic cusp; (e) sepsis and (f) contraindication to antiplatelet therapy.

Percutaneous closure of a VSD was performed under general anesthesia. Femoral vein and arterial line were obtained. For procedure we gave $100 \mathrm{IU} / \mathrm{kg}$ heparin in two divided doses (50 IU/kg at first and $50 \mathrm{IU} / \mathrm{kg}$ half an hour later) for each patient to maintain activated clotting time longer than 200 sec after obtaining the femoral artery. Intravenous antibiotic with $30 \mathrm{mg} / \mathrm{kg}$ Cefazoline was administered prophylactically at beginning of the procedure and two subsequent doses 8 hours during the following 24 hours. The procedure was performed under fluoroscopic and transthoracic echocardiographic (TTE) control. Standard right and left cardiac catheterization and angiography were performed and data was gathered. During the cardiac procedure angiographic and catheterization data including size and type of VSD, its relation to aorta and being of an aneurysmal formation of VSD were obtained from the ventriculogram in left-anterior-oblique (LAO) in different degree's and also rightanterior-oblique (RAO) views for relation by pulmonary artery (Figure 1).

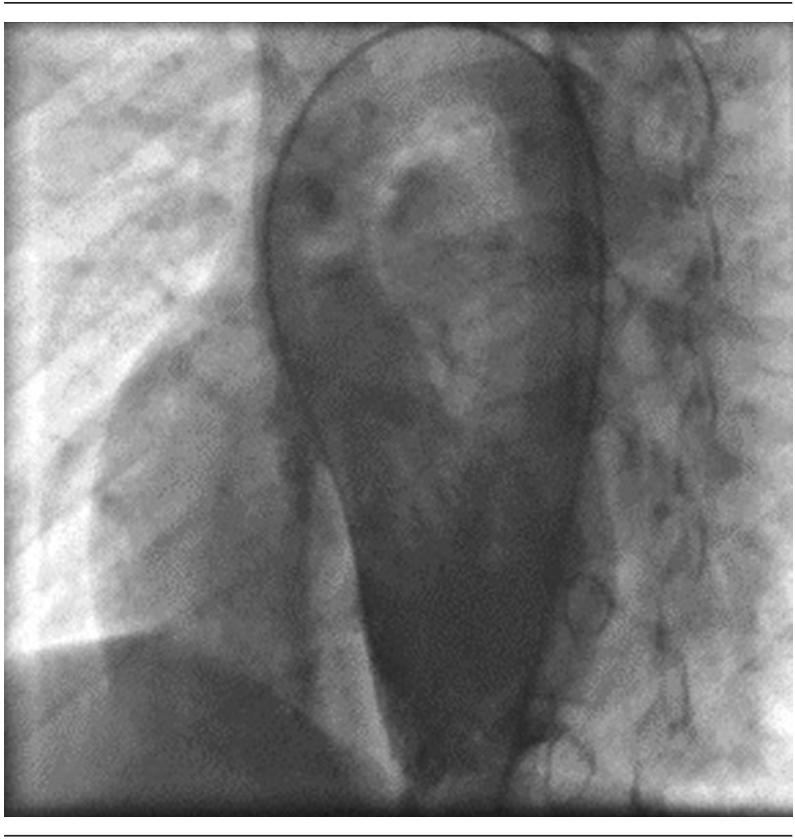

Figure 1. Left Ventriculogram at the Beginning of Procedure for Estimation of Perimembrous VSD Size and Relation to Aortic Valve

LAO aortogram in ascending aorta was obtained for identifying of aortic cusp prolapse. Right and left ventricular pressures, pulmonary artery pressure and fluoroscopy time were identified via right and left cardiac study. Total time of procedure was estimated from beginning to the end of procedure. The diameter of pulmonic end of 
chosen ADO was at least $2 \mathrm{~mm}$ larger than the narrowest of the measured VSD diameter in right side by ventriculogram. Subsequently, a 0.035 inch Terumo glide wire was placed across the VSD using a 4 or 5 French curved endhole catheter (Judkins right coronary, Cobra) or manually cutting a pig-tail catheter to adjust the needed angle from the left ventricle (LV) into either branch of the pulmonary artery, and superior or inferior vena cava. At the next step the wire was snaring with Amplatz Gooseneck Snare and withdrawn to exteriorize it through femoral vein and establishing a long wire loop from the femoral artery to the femoral vein. Over this wire, an appropriate size dilator and long sheath was advanced from the femoral vein across the VSD by standard protocol all the way until the tip of the sheath was in the descending aorta (Figure 2).

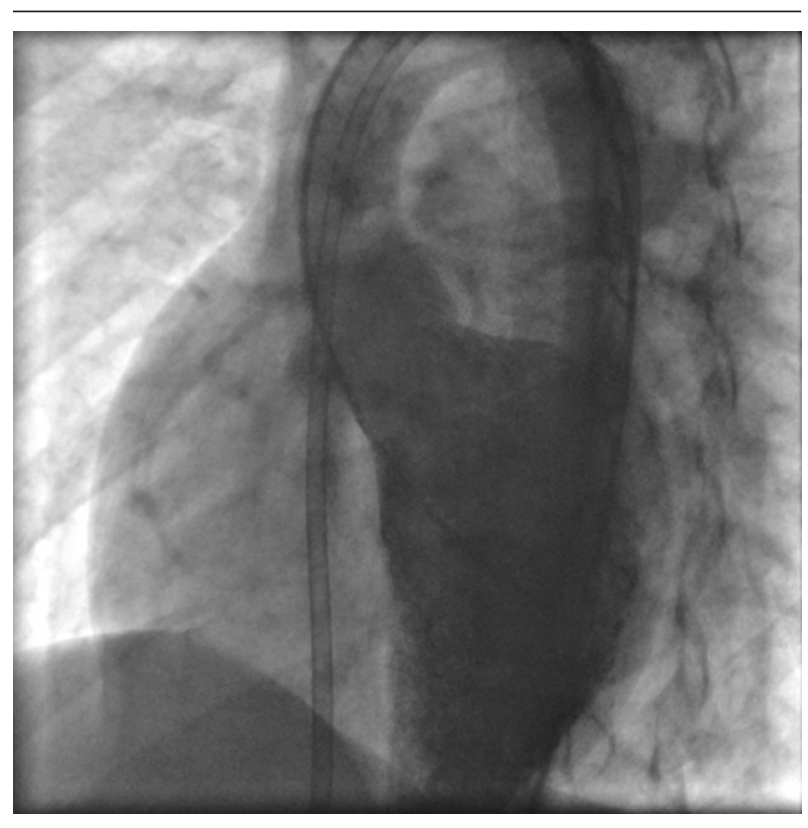

Figure 2. Left Ventriculogram After Advancing of an Appropriately Sized Sheath From the Femoral Vein Across the VSD

Then the dilator was removed from the vein line and guide wire and end-hole catheter were removed from arterial line. At this time for measuring of VSD diameter ventriculogram was repeated. After flushing the long sheath, the device was loaded under a blood/saline solution and the device attached to the delivery sheath and advanced to the tip of the sheath during fluoroscopy. At this time the retention disc of the ADO device was deployed and following which the cable delivery sheath and device were pulled as one unit under fluoroscopy until the retention disk was positioned at the left ventricular side of VSD not interfering with aortic valve cusps. At this stage left ventriculography and echocardiography were performed to confirm appropriate device position and aortogram to confirm absence of aortic regurgitation. The tubular frame of the ADO device was deployed by pulling the sheath in to the right ventricle. The device was released by turning the cable after confirmation of good device position. Aortogram and left ventriculogram were taken 5 - 10 minutes after releasing the device (Figure 3). Chest X ray (CXR), complete blood cell counts (CBC) and echocardiography were done 4-6 hours later for early complication such as occult hemorrhage, pulmonary complication and pericardial effusion. Urine analysis for exclusion of hemolysis was done the day after procedure. Vital signs monitoring was done during first 24 hours. The patients were monitored with electrocardiogram for 24 hours. 12 -lead electrocardiogram was obtained one day, one month, three months and yearly after the device implantation. Echocardiography was performed one day, one month, three months, 12 months and yearly after discharge. All patients received Clopidogrel $1 \mathrm{mg} / \mathrm{kg}$ for three days and $0.5 \mathrm{mg} / \mathrm{kg}$ for one month, acetyl salislic acid (ASA) 3-5 mg/kg for six months and heparin $50 \mathrm{IU} / \mathrm{kg}$ every six hours for first day by control of clotting tests. All patients were discharged 24 hours after procedure. Endocarditis prophylaxis was done for first six months if needed and discontinued at the six month's follow-up by echocardiography if the defect was completely occluded and had no residue. Data are expressed as a frequency or percentage for nominal variables. Continuous variables are expressed as Mean \pm standard deviation (SD). Analyses were performed by using SPSS Statistics 18.0. Statistical significance was defined as a P value $<0.05$.

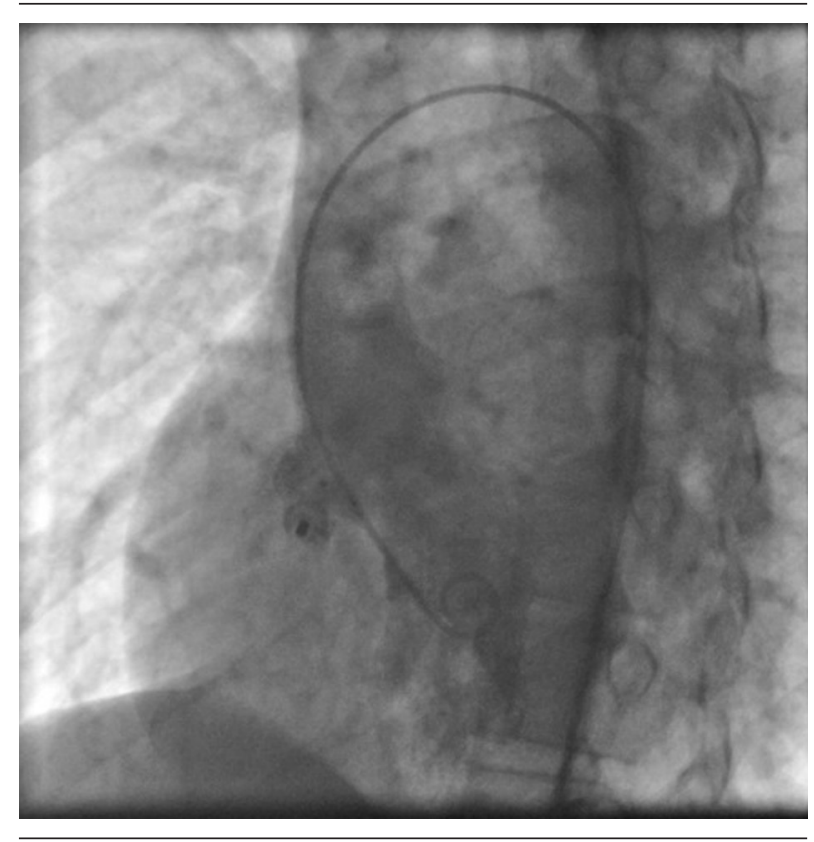

Figure 3. Left Ventriculogram After Releasing of ADO in the Perimembranous VSD Shows Well Positioned ADO in Aneurysm of VSD

\section{Results}

During the period of the study, with the intention to close the VSD, 28 patients underwent transcatheter closure of VSD between April 2012 and April 2013. Mean 
duration of follow-up was $8.3 \pm 3.6$ months (range 1 to 18 months). We had 16 female and 12 male patients. Table 1 shows demographic, general, clinical, and analytical data of the patients. All 28 patients showed LV enlargement in echocardiographic examination. Fifteen patients showed PMVSD with aneurysm. The defects were successfully closed in $27 / 28$ (96.4\%) patients. We had no mortality or major arterial complications such as massive bleeding or femoral arteriovenous fistula. Hemolysis did not happen in any patient. Mild thrombosis of right or left femoral artery and reducing arterial pulse occurred in 4 patients. Arterial surgical consult and Doppler sonographic study were done for all these patients. Intravenous infusion of heparin produced a successful and complete turn of pulse without sequel. No patient needed femoral artery thrombectomy. Device embolization did not occur in any patient. No aortic valve regurgitation was observed in our patients. In five patients, transient arrhythmia such as tachycardia and bradycardia was seen during different maneuvers of the catheter or guide wire. As the procedure stopped, sinus rhythm recovered quickly. Transient AVB was found in one patient during different maneuvers of the catheter or guide wire during procedure, but disappeared quickly without any management, so the procedure was continued to implant the device successfully. After discharge of hospital during follow-up no deaths or cases of infectious endocarditis occurred in our patients. In cases with aneurysmal PMVSD we tried to occlude larger hole in left ventricle with Amplatzer but in two patients Amplatzers were implanted in aneurysmal sac and in one of these patients we had mild residual shunt (by echocardiographic evidence) during follow-up. Ventricolography at the end of the procedure demonstrated that complete occlusion rate was $65.7 \% \mathrm{im}$ mediately after completion of the procedure, rising up to $79.5 \%$ at discharge and $96.4 \%$ during follow-up. The complete heart block did not happen during the follow-up pe- riod. Follow up to this time (1 month - 18 months) showed no significant complications such as device embolization or malposition, thrombus or clot formation, hemolysis or thromboembolism.

\section{Discussion}

This study reports results and follow-up findings in 28 children who underwent transcatheter closure of VSD using Amplatzer PDA. Transcatheter closure of VSD like other interventional approaches has complications such as femoral artery thrombosis and arterial complications, hemolysis and embolization of the device. However, the high incidence of complete atrioventricular block (CAVB) after device closure of a PMVSD has been a serious and worrisome complication (10-15). The CAVB rates reported in most reports is around 5\% of CAVB. Predescu reported higher incidence $(8,9,12)$. In patients treated percutaneously for closing VSD Butera et al reported that the occurrence of CAVB compared with surgical closing is a late complication, CAVB usually appears soon after the surgery (11). There are no definite data about the mechanisms responsible for the occurrence of CAVB after percutaneous closure of a PMVSD. This complication has been reported to occur within a few days to months after successful and uncomplicated procedure (16-18). It is suggested that occurrence of CAVB after interventional closure of PMVSD with APMVSDO is caused by an oversize device through direct compression and traumatizing of the surrounding tissue that provokes inflammatory reaction or scar formation and induces CAVB in conductive tissue (10). The occurrence of CAVB is mostly related to the anatomy of conduction system in the heart and around the margins of the defect, so both surgical and interventional approaches could induce complete heart block. By pointing out the anatomy of conduction pathway can be understood what causes such a high rate of CAVB. After originating of His bundle from atrioventricular node and

\begin{tabular}{|c|c|c|c|c|}
\hline Variant & Total & Male & Female & Range (Total) \\
\hline Age, $y$ & $4.7 \pm 6.3$ & $5.1 \pm 6.2$ & $4.4 \pm 4.6$ & $2-14$ \\
\hline Weight, kg & $14.7 \pm 10.5$ & $15.8 \pm 14.4$ & $14.1 \pm 12.2$ & $10-40$ \\
\hline VSD size at right ventricle, $\mathrm{mm}$ & $4.5 \pm 1.6$ & $4.4 \pm 1.5$ & $4.7 \pm 1.6$ & $2.8-9.2$ \\
\hline \multicolumn{5}{|l|}{ PA pressure } \\
\hline Systolic & $32.9 \pm 17.2$ & $35.2 \pm 18.1$ & $31.2 \pm 11.1$ & $18-52$ \\
\hline Diastolic & $15.7 \pm 8.1$ & $17.1 \pm 8.9$ & $15.1 \pm 7.6$ & $12-39$ \\
\hline Mean PA pressure, $\mathrm{mmHg}$ & $21.7 \pm 6.2$ & $24.2 \pm 6.8$ & $20.1 \pm 5.5$ & $12-37$ \\
\hline Fluoroscopy time, $\min$ & $15.4 \pm 8.7$ & $14.1 \pm 12.1$ & $15.9 \pm 8.7$ & $9.7-38.5$ \\
\hline Total angiography time, min & $43 \pm 14.3$ & $41.5 \pm 11.6$ & $43.9 \pm 12.9$ & $38-80$ \\
\hline Size of Amplatzer (narrowest size at right ventricle), mm & $7.3 \pm 3.2$ & $7.6 \pm 3.1$ & $6.4 \pm 2.9$ & $4-12$ \\
\hline
\end{tabular}


piercing the tricuspid valve (TV) annulus, it reaches ventricular septum and goes along the posteroinferior margin of the membranous septum. Then it divides into left bundle branch (LBB) and right bundle branch (RBB) and these divide further into smaller branches. Left bundle branch passes through the ventricular septum and right bundle branch through intramyocardium. By using Amplatzer, the ventricular septum which has a conduction pathway, could be squeezed by the two discs of the device, and as time passes, fibrotic changes occur between the device and the septum and CAVB may occur. In addition, as the device is too stiff and especially if an oversized device was chosen, it could facilitate more traumatic and inflammatory reaction in the conductive tissue.

For these reasons we used ADOs that have shorter distal rim than other devices and have not a proximal disc, thus it does not squeeze His bundle and causes less CAVB. Lee et al. used ADO for closing PMVSD and they had no CAVB in their patients (19). We had also no CAVB in our patients immediately and during follow-up. Transient AVB was found in one patient during procedure because of different maneuvers of the catheter, but disappeared quickly without any management and the procedure was continued successfully. In our series, the median follow-up period was 8 , ( $3 \pm 3.6$ months range 1 to 18 months) and CAVB was not seen in any patient; a long term follow-up is needed for more evaluation.

Some studies reported initial successful reversal or prevention of CAVB using steroids but one study reported of reappearance of complete atrioventricular block requiring a definitive pacemaker implantation after an initial successful reversal using both steroids and aspirin (10, 20,21 ). We did not use steroids for prevention of pacemaker implantation in our patients. Complete AVB may occur in completely asymptomatic and healthy patients and CAVB should be monitored as a late event occurring in post procedural phases during follow-up. The occurrence of CAVB was completely unpredictable and some patients appeared years after the device closure (22). Percutaneous closure of PMVSD in young children must be carefully suggested because CAVB occurred mostly in patients with less than five years old at the time of the procedure. In follow-up we monitor our patients with routine twelve lead ECG in every visit and if needed by 24 or 48 hours ECG holter monitoring. However, in our study no early and mid-term major complication such as mortality, CAVB, device emboli, arteriovenous fistula, hemolysis and endocarditis occurred. This study confirmed effectiveness of transcatheter closure of PMVSD using ADO during follow up. A limitation of our study was its mid-term follow-up duration.

Transcatheter closure of VSD with ADO in symptomatic infants is an effective and safe method for the treatment of medium to large VSD. The low incidence of complications such as CAVB and short hospital stay makes ADO ideal for transcatheter closure of medium to large VSD in children. For evaluation of this method long term followup and more study with larger number of patients is suggested.

\section{References}

1. The natural history of ventricular septal defects in infancy. Hoffman JI, Rudolph AM. Am J Cardiol. 1965;16(5):634.

2. Current expectations for surgical repair of isolated ventricular septal defects. Scully BB, Morales DLS, Zafar F, McKenzie ED, Fraser DC, Heinle JS. Ann Thorac Surg. 2010;89(2):544.

3. High prevalence of muscular ventricular septal defect in neonates. Roguin N, Du ZD, Barak M, Nasser N, Hershkowitz S, Milgram E. J Am Coll Cardiol. 1995;26(6):1545.

4. Effect of technical development on the apparent incidence of congenital heart disease. Tikanoja T. Pediatr Cardiol. 1995;16(2):100.

5. New device for closure of muscular ventricular septal defects in a canine model. Amin Z, Gu X, Berry JM, Bass JL, Titus JL, Urness M, et al. Circulation.1999;100(3):320.

6. Transcatheter closure of muscular ventricular septal defects with the amplatzer ventricular septal defect occluder: initial clinical applications in children. Thanopoulos BD, Tsaousis GS, Konstadopoulou GN, Zarayelyan AG. J Am Coll Cardiol. 1999;33(5):1395.

7. Initial human experience with the Amplatzer perimembranous ventricular septal occluder device. Bass JL, Kalra GS, Arora R, Masura J, Gavora P, Thanopoulos BD, et al. Catheter Cardiovasc Interv. 2003;58(2):238

8. Percutaneous closure of perimembranous ventricular septal defects with the Amplatzer device: technical and morphological considerations. Pedra CA, Pedra SR, Esteves CA, Pontes SJ, Braga SL, Arrieta SR, et al. Catheter Cardiovasc Interv. 2004;61(3):403.

9. Transcatheter closure of perimembranous ventricular septal defects using amplatzer asymmetric ventricular septal defect occluder: preliminary experience with 18-month follow up. Pinto RJ, Dalvi BV, Sharma S. Catheter Cardiovasc Interv. 2006;68(1):145.

10. Atrioventricular block after transcatheter closure of perimembranous ventricular septal defects. Walsh MA, Bialkowski J, Szkutnik M, Pawelec-Wojtalik M, Bobkowski W, Walsh KP. Heart. 2006;92(9):1295.

11. Transcatheter closure of perimembranous ventricular septal defects: early and long-term results. Butera G, Carminati M, Chessa M, Piazza L, Micheletti A, Negura DG, et al. J Am Coll Cardiol. 2007;50(12):1189.

12. Complete heart block associated with device closure of perimembranous ventricular septal defects. Predescu D, Chaturvedi RR, Friedberg MK, Benson LN, Ozawa A, Lee KJ. J Thorac Cardiovasc Surg. 2008;136(5):1223.

13. Late complete atriovenous block after percutaneous closure of a perimembranous ventricular septal defect. Butera G, Massimo C, Mario C. Catheter Cardiovasc Interv. 2006;67(6):938.

14. Early and delayed atrioventricular conduction block after routine surgery for congenital heart disease. Lin A, Mahle WT, Frias PA, Fischbach PS, Kogon BE, Kanter KR, et al. J Thorac Cardiovasc Surg. 2010;140(1):158.

15. Transcatheter closure of perimembranous ventricular septal defect: is the risk of heart block too high a price? Sullivan ID. Heart. 2007;93(3):284

16. Transcatheter closure of perimembranous ventricular septal defects using the amplatzer membranous VSD occluder: immediate and midterm results of an international registry. Holzer R, de Giovanni J, Walsh KP, Tometzki A, Goh T, Hakim F, et al. Catheter Cardiovasc Interv. 2006;68(4):620.

17. Heart block and empirical therapy after transcatheter closure of perimembranous ventricular septal defect. Yip WC, Zimmerman F, Hijazi ZM. Catheter Cardiovasc Interv. 2005;66(3):436.

18. Percutaneous closure of perimembranous ventricular septal defects with Amplatzer occluders--a single centre experience. Szkutnik M, Kusa J, Bialkowski J. Kardiol Pol. 2008;66(9):941. discussion 948-9.

19. Transcatheter closure of perimembranous ventricular septal 


\section{Ghaderian Met al.}

defect using Amplatzer ductal occluder. Lee SM, Song JY, Cho JY, Lee SY, Paik JS, Chang SI, et al. Catheter Cardiovasc Interv. 2013;82(7):1141.

20. Is steroid therapy enough to reverse complete atrioventricular block after percutaneous perimembranous ventricular septal defect closure? Butera G, Gaio G, Carminati M. J Cardiovasc Med (Hagerstown). 2009;10(5):412.
21. Temporary atrioventricular complete block that develops following the transcatheter closure of ventricular septal defect. Erdem S, Kizlltas A, Kucukosmanoglu O, Ozbarlas N. Turk J Pediatr. 2012;54(1):80.

22. Late high degree atrioventricular block after percutaneous closure of a perimembranous ventricular septal defect. Yalonetsky S, Lorber A. Cardiol Young. 2009;19(3):298 